\title{
NOVA OUTBURST DUE TO ACCRETION OF H-RICH MATTER ONTO WHITE DWARFS
}

G. Siegfried Kutter*

and

Warren M. Sparks

NASA/Goddard Space Flight Center

Laboratory for Astronomy and Solar Physics

Greenbelt, Maryland 20771

\section{Introduction}

We assume that the outburst of classical novae is the result of transfer of H-rich material from a red secondary star to a He or $\mathrm{C} / \mathrm{O}$ white dwarf and the development of a thermonuclear runaway in the edegenerate base of the accreted H-rich envelope. Based on these assumptions, we have investigated this problem in several stages of increasing theoretical complexity and physical realism.

In the first stage we neglect the process of accretion and assume that the H-rich material is already on the white dwarf and in hydrostatic and thermal equilibrium (Sparks, Starrfield, and Truran 1978, and references given therein). Prialnik, Shara, and Shaviv (1978) have also investigated this stage.

In the second and third stages, we take the process of accretion into account. In stage two, we make the simplifying assumption that the H-rich material arrives on the white dwarf in a spherically symmetric manner. This stage has also been investigated by Nariai, Nomoto, and Sugimoto (1979). In stage three, we drop the restriction of spherical symmetry in the accretion and assume that the hydrogen arrives with angular momentum, as it does when it is accreted from a circumstellar disk. We do not take into account the effects of either the accretion disk or of the secondary star.

The present report deals with stage two, and the following report (Sparks and Kutter, page 294 ) deals with stage three.

* On leave of absence from The Evergreen State College, Olympia, WA 
II. Definition of Stellar Model and of Computing Technique

$10^{-3}$ Our stellar model is a I $\mathrm{M}$ He white dwarf with a luminosity of

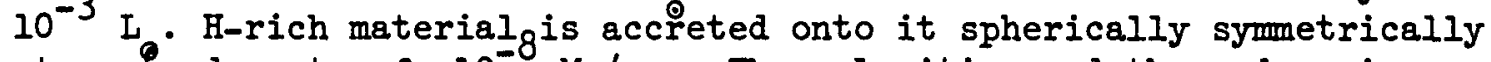
at a steady rate of $10^{-8} \mathrm{M} / \mathrm{yr}$. The velocities and thermodynamic conditions of the arriving hydrogen are assumed to be identical to those of the accreted H-rich envelope's outermost mass zone. The composition of the H-rich material is $X_{H}=0.7306, X_{H e}=0.2494, X_{C}=0.0034$, $\mathrm{X}_{\mathrm{N}}=0.0013, \mathrm{X}_{\mathrm{O}^{\prime}}=0.0082$.

our computing is done with the implicit hydrodynamic code that formed the basis of the first stage of our investigation (Kutter and Sparks, 1972), except for modifications to include mass accretion. The nuclear reaction rates and all other auxiliary functions are computed identically to those in the earlier work.

\section{Results}

our results are most concisely summarized by the following graph, in which we show the evolution of the maximum temperature attained in the H-rich envelope versus time:

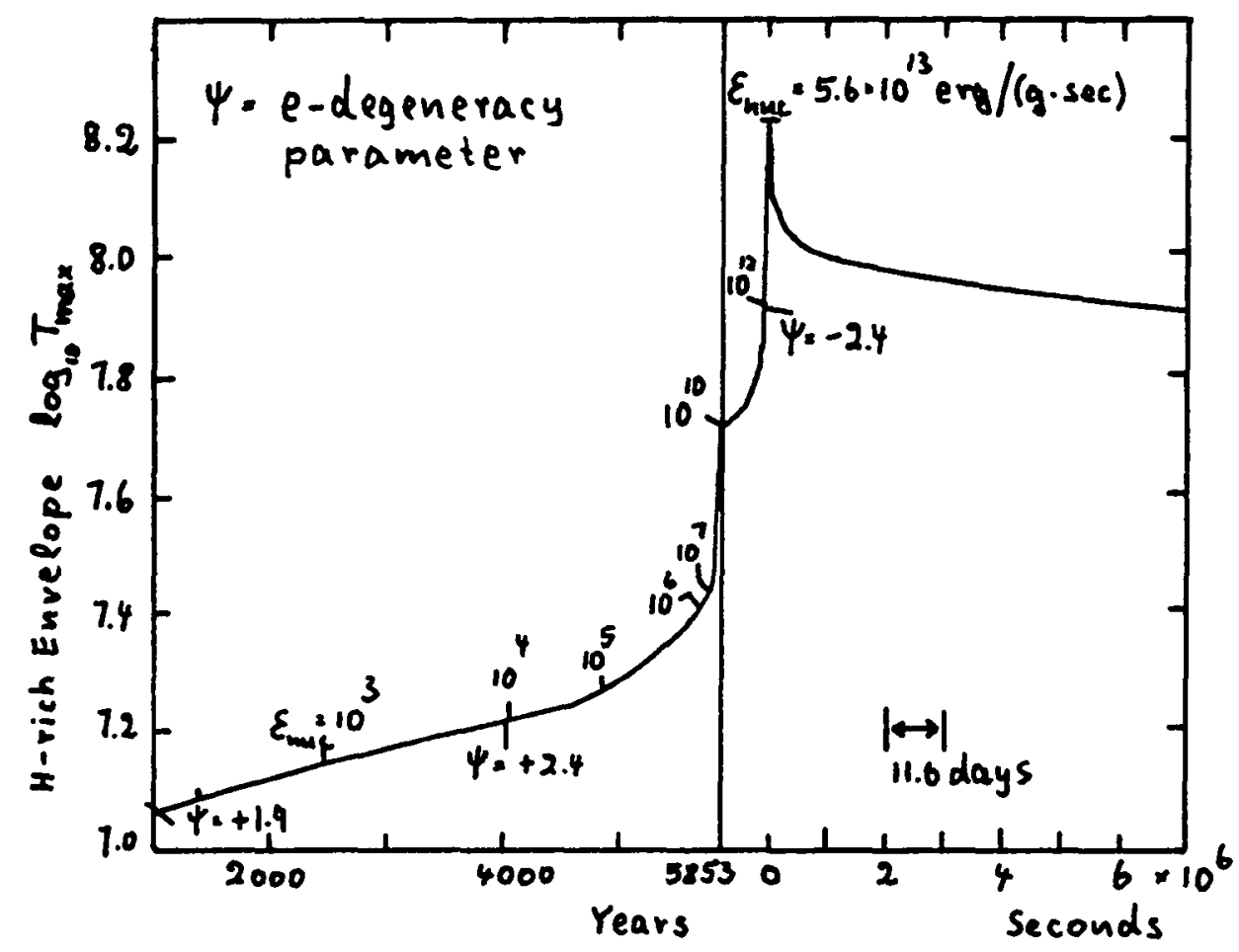


The white dwarf accretes H-rich material for 5848 years. By then the thermonuclear runaway is well under way, and the time scale of evolution of the H-rich envelope has become so short that further accretion is unimportant. We, therefore, turn accretion off. Just 5 years later, at age 5853 years, the runaway peaks.

During the first 5000 years of accretion, the energy balance in the H-rich envelope is totally dominated by the gravitational energy released by accretion. This causes the uniform increase in the maximum temperature of the envelope shown in the graph.

From 5000 years on, the energy released by the nuclear reactions near the base of the H-rich envelope dominates the energy balance more and more. The maximum temperature of the envelope rises exponentially, reaching a peak of 8.220 in $\log _{\text {at age }} 5853$ years ( age 0 seconds on the new time scale, right half of graph). Along with this increase in temperature, the rate of nuclêr energy release, $\varepsilon_{\text {nuc }}$ also rises, reaching a peak value of $5.6 \cdot 10^{13} \mathrm{erg} /(\mathrm{g} \cdot \mathrm{sec})$.

Although the peak value of $\varepsilon_{\text {nuc }}$ is large by most standards of stellar evolution, our experiences from the first stage of our investigation indicate that it is too low by about 2 orders of magnitude for mass ejection typical of fast novae. Our present numerical results confirm this. For instance, 55 days after peak burning (when the surface mass zones of the envelope are just beginning to become optically thin), the structure of the H-rich envelope is characterized as follows:

$$
\begin{aligned}
& E_{\text {grav }}=-6.3(45) \mathrm{erg}, \\
& E_{\text {therm }}=4.3(45) \mathrm{erg}, \\
& E_{\text {kin }}=1.4(36) \mathrm{erg} .
\end{aligned}
$$

Evidently, the H-rich envelope still sits deep in the gravitational well of the star, and its kinetic energy is negligible. Further, the temperature and nuclear energy generation rates are dropping, and the outward expansion is decelerating. Nova-like mass ejection does not occur.

The reason for this limited release of nuclear energy and lack of mass ejection lies with the accretion itself. The gravitational energy released by accretion keeps the e-degeneracy of the base of the H-rich envelope at moderate levels. As we have indicated in the graph, the e-degeneracy parameter (chemical potential/kT) rises only to about +2.4 at age 4000 years and then diminishes again. This lack of strong edegeneracy limits the strength of the nuclear runaway and, consequently, prevents mass ejection. In contrast, from our stage-one work, we know that the e-degeneracy parameter should be approximately +10 or greater for nova-producing runaways. 
We would like to emphasize that for mass accretion rates considerably lower than ours, there would be sufficient time to radiate away much of the gravitational energy released. Consequently, the e-degeneracy and the runaway would be stronger, and mass ejection would be more likely. In fact, for accretion rates lower than $10^{-10} \mathrm{M}_{\odot} / \mathrm{yr}, \mathrm{M} / \dot{\mathrm{M}}$ is greater than the time scale of energy diffusion through ${ }^{\circ}$ the $\mathrm{H}-\mathrm{r}_{\mathrm{I}} \mathrm{ch}$ envelope; and the structure of the accreting envelope should closely resemble the envelopes of our stage-one investigation.

\section{Conclusion}

In the present, stage-two investigation we find that accretion of $H$-rich material onto a heavy-element $I M$ white dwarf under the assumptions of spherical symmetry and of ${ }^{\circ}$ moderate accretion rates (like $10^{-8} \mathrm{M} / \mathrm{yr}$ ) does not produce nova-like mass ejection. We doubt that our assumed accretion rate or the mass chosen for the white dwarf are unphysical in modelling the nova outburst. However, we do believe that our assumption of spherical symetry for the accretion process is too idealized.

We, therefore, conclude that we must take into account the angular momentum that the H-rich material possesses as it arrives on the white dwarf. If the rotational velocities associated with this angular momentum are approximately Keplerian, large shears are set up between the accreting H-rich material and the white dwarf; turbulent mixing and penetration of the H-rich material into the white dwarf occurs; and the temperature and composition structures of the envelope become greatly altered. This, in turn, strongly affects the physical details of the outburst. Our stage-three investigation deals with this more realistic kind of modelling of the classical nova outburst (Sparks and Kutter, page 294).

\section{References}

Kutter, G. S., and Sparks, W. M. 1972, Ap. J., 175, 407.

Nariai, K., Nomoto, K., and Sugimoto, D. 1979, submitted to Publ. Astr. Soc. Japan.

Prialnik, D., Shara, M. M., and Shaviv, G. 1978, Astr. Ap., 62, 339.

Sparks, W. M., Starrfield, S., and Truran, J. W. 1978, Ap. J., 220, 1063. 